msh-mss Mathématiques et sciences humaines

159 | Automne 2002

Varia

\title{
Modéliser les activités cognitives des hommes au Paléolithique
}

A model of paleolithic man cognitive abilities

Isabelle Saillot, Marylène Patou-mathis, Jean-François Richard, Emmanuel Sander et Sébastien Poitrenaud

\section{(2) OpenEdition}

12 Journals

Édition électronique

URL : http://journals.openedition.org/msh/2866

DOI : $10.4000 / \mathrm{msh} .2866$

ISSN : 1950-6821

Éditeur

Centre d'analyse et de mathématique sociales de l'EHESS

Édition imprimée

Date de publication : 1 septembre 2002

ISSN : 0987-6936

Référence électronique

Isabelle Saillot, Marylène Patou-mathis, Jean-François Richard, Emmanuel Sander et Sébastien

Poitrenaud, "Modéliser les activités cognitives des hommes au Paléolithique », Mathématiques et

sciences humaines [En ligne], 159 | Automne 2002, mis en ligne le 10 février 2006, consulté le 23 juillet 2020. URL : http://journals.openedition.org/msh/2866 ; DOI : https://doi.org/10.4000/msh.2866 


\title{
MODÉLISER LES ACTIVITÉS COGNITIVES DES HOMMES AU PALÉOLITHIQUE
}

\author{
Isabelle SAILLOT ${ }^{1}$, Marylène PATOU-MATHIS ${ }^{1}$, \\ Jean-François RICHARD², Emmanuel SANDER ${ }^{2}$, Sébastien POITRENAUD ${ }^{2}$
}

\begin{abstract}
RÉSUMÉ - La plupart des recherches sur la cognition au Paléolithique utilisent un vocabulaire et une méthodologie ad hoc, ou encore font appel à des concepts de psychologie en leur ôtant leur signification d'origine. Aucune discussion ne peut donc déboucher sur un consensus. Dans cet article, nous proposons une approche qui consiste à employer certains des concepts développés puis utilisés depuis plusieurs années par la psychologie cognitive, et plus particulièrement l'une de ses branches expérimentales. La psychologie des activités mentales finalisées semble en effet très bien adaptée à la modélisation du comportement humain et des capacités cognitives associées, au Paléolithique. Nous montrons ici comment ces outils conceptuels, développés pour la cognition contemporaine, peuvent néanmoins être utilisés par le préhistorien, dans les conditions d'une expérimentation en laboratoire. Cette approche pourrait fournir des descriptions rigoureuses de certaines caractéristiques cognitives anciennes, en particulier en ce qui concerne la catégorisation, un mécanisme partagé par tous les primates. Des résultats préliminaires concernant le niveau Magdalénien de la grotte Tournal sont présentés et discutés.
\end{abstract}

MOTS-CLÉS - Palethnologie, Paléo-psychologie expérimentale, Catégorisation.

SUMMARY - A model of paleolithic man cognitive abilities

As far as paleolithic cognitive processes are concerned, most studies define their own vocabulary and method, usually using current words while ascribing them various meanings. No debate can hence be fruitfull. We suggest an alternative approach which is to address the numerous and accurate concepts developped since about fourty years by cognitive psychology and especially its experimental branch. Cognitive psychology of goal oriented actions is in fact a powerful tool provider in the attempt to understand and modelize paleolithic men behaviour and cognitive abilities. Here we show why and how it is possible to use these tools initially developped to investigate modern man cognition in the laboratory conditions. We claim that the cognitive psychology method presented in this article allows to draw a rigorous description of mental organization, especially about the complex cognitive process of memory and categorization, whatever human species is dealt with and wathever paleolithic period is concerned. A preliminary result concerning the Magdalenian level of the Tournal cave is produced and discussed.

KEYWORDS - Paleolithic behaviour, Experimental paleo-psychology, Categorization.

\footnotetext{
${ }^{1}$ Laboratoire de Préhistoire du Muséum National d'Histoire Naturelle de Paris, UMR 9948 du CNRS, Institut de Paléontologie humaine, 1 rue René Panhard 75013 Paris, e-mail : saillot@mnhn.fr, patmath@mnhn.fr.

${ }^{2}$ Laboratoire de Psychologie cognitive, ESA CNRS 7021, Université Paris 8 Saint-Denis, 93326 SaintDenis, e-mail : richard@univ-paris8.fr, sander@univ-paris8.fr, poitrenaud@univ-paris8.fr.
} 
Nous proposons ici une méthode de modélisation de la catégorisation des hommes du paléolithique, ainsi qu'une application de cette méthode à un site du paléolithique supérieur. Le résultat obtenu est un réseau sémantique des actions, graphe de la catégorisation associée à diverses tâches sur le site, dont nous suggérons une description et une interprétation. Nous nous plaçons dans la perspective cognitive de J.-F. Richard [1990], appliquée à des données du comportement de subsistance [M. Patou-Mathis, 1996].

Dans les études sur les processus cognitifs au paléolithique, les auteurs ont jusqu'à présent cherché à forger un vocabulaire ad hoc. Les notions et concepts mis en place depuis plusieurs décennies par la psychologie cognitive n'ont pratiquement jamais été utilisés. La psychologie cognitive des actions finalisées semble pourtant fournir des outils très performants pour modéliser le comportement paléolithique.

\section{UN DOMAINE CONFUS}

A. Belfer-Cohen et N. Goren-Inbar [1994] rappellent judicieusement qu'une cause importante de confusion est l'emploi généralisé de termes identiques aux acceptions différentes pour chaque auteur, par exemple "planification », qui revêt des réalités disjointes entre T. Wynn [1979, 1981] et S.L. Kuhn [1992]. En effet, T. Wynn entend ce terme au sens d'organisation d'une séquence d'actes aboutissant à une action (taille d'outils, découpe de gibier, par exemple). Pour S.L. Kuhn, la planification est une prévision d'actions à bien plus long terme, d'une saison sur l'autre par exemple (stokage de nourriture, anticipation des migrations des animaux, ...). Ces deux acceptions sont donc tout à fait incompatibles.

J. Gervet \& al. [1996] signalent que l'acception du terme « représentation » varie avec chaque auteur. Ils proposent d'abandonner ce terme, et de considérer à la place des « images d'actes » qui expriment l'état de l'organisme quand la chaîne sensorimotrice concernée est mise en action. Or, la notion de représentation est probablement la plus cruciale du domaine des sciences cognitives.

En effet, il existe deux types de constructions mentales : les représentations et les connaissances. Les constructions mentales regroupent l'ensemble des informations prises en compte par le système cognitif dans la réalisation de la tâche. Les connaissances peuvent être définies comme des constructions stables, stockées en mémoire à long terme, alors que les représentations seraient des constructions circonstancielles, faites dans un contexte particulier et intimement lié à lui. Le système cognitif dispose de différentes fonctions, et peut réaliser différentes classes d'activités. La construction des représentations est la fonction correspondant à la classe d'activités de compréhension. Autrement dit, le produit de la compréhension est une représentation de la situation.

On voit donc qu'en psychologie cognitive, la notion de représentation ordonne tout l'édifice théorique en conférant leurs significations et leurs relations mutuelles aux 
concepts principaux du modèle, tels que : connaissance, information, tâche, mémoire, fonction.

Pour le préhistorien, la notion de représentation est opératoire et bien circonscrite dans son domaine d'origine, il convient donc de l'exploiter judicieusement, en aucun cas de lui substituer une notion intuitive sans contenu scientifique.

R. Bednarik [1995] ajoute de la confusion au débat. Il s'appuie sur l'étude des sites de Bilzingsleben et de Tata, qui auraient livré des pièces gravées, hypothèse depuis réfutée par l'un des auteurs, pour soutenir que l'expression symbolique et les capacités cognitives associées étaient présentes au paléolithique inférieur et moyen. Les termes d'intentionnalité et de catégorisation sont employés sans référence. En sciences cognitives, l'intentionnalité n'est pas le «caractère intentionnel» d'une production artistique par exemple, mais « la capacité représentative des états mentaux..., le fait qu'ils soient à propos de certains objets » [J.-P. Dupuy, 1994]. La question de l'origine de l'intentionnalité, en ce sens, est l'une des plus débattue par les psychologues, notamment autour du débat sur sa «naturalisation ». Ces réflexions ont contribué à asseoir quelques consensus, dont le préhistorien peut tirer un bénéfice immédiat: méconnaître la fonctionnalité ou pire, la définition même, de cette notion cruciale est à notre sens une erreur fondamentale du paléo-psychologue.

De même, il n'existe aucun lien simple entre la notion cognitive de catégorisation et le fait que l'homme forme occasionnellement des collections d'objets. La catégorisation est la capacité d'organiser nos connaissances selon des concepts et des classes de concepts. Elle consiste d'une part «à délimiter la classe, et d'autre part à définir les relations entre cette classe et les autres »[J.-F. Richard, 1990]. Il est impossible de ne pas considérer la confusion entre le processus de catégorisation et l'activité de collecte (ou collection) comme relevant d'un contresens élémentaire. Nous verrons plus loin comment les processus de catégorisations sont à notre sens les plus importants, actuellement, pour le préhistorien, car les plus directement accessibles à l'aide de cette nouvelle méthode.

W. Noble, I. Davidson [1991] utilisent l'adjectif « symbolique » dans un sens très personnel, sans le préciser. Pour les auteurs, ce qui est spécifiquement humain, c'est l'utilisation de symboles. Les auteurs reprochent à des théories non centrées sur les symboles d'être intrinsèquement spéculatives. Pour les auteurs, un comportement est symbolique si et seulement si il conduit au tracé de symboles sur un support. En optant pour une acception moins étroite du symbole, telle que la formule la linguistique, ainsi que le soulignent O. Ducrot et T. Todorov [1972], il est possible de donner du comportement symbolique la définition qu'adopte J.-F. Richard [1990], et que nous reprenons à notre compte ici, à savoir une «construction de représentations, raisonnements, élaboration de stratégies... ». Les pratiques de ces auteurs prouvent que la définition ad hoc de concepts cognitifs par les préhistoriens brouille irrémédiablement la recherche dans le domaine [I. Saillot, M. Otte, M. Patou-Mathis, 2000-2001]. 


\section{DES CAPACITÉS COGNITIVES MODERNES}

Pour de nombreux auteurs, la cognition des paléolithiques est très proche de la nôtre. Pour M. Otte [1988], « le comportement de l'homme au Paléolithique moyen apparaît remarquable dans la subtilité de son adaptation », et «toutes les possibilités évolutives existaient dès le Moustérien, qui les possédait donc en puissance ». Il en est de même pour M. Otte, M. Patou-Mathis [1992], selon qui l'homme de Néandertal révélerait «...une complexité comportementale telle que l'on peut déjà parler de sociétés de type moderne ». J. Pelegrin \& al. [1988] et J. Pelegrin [1993] analysent les chaînes opératoires lithiques dans l'optique cognitive et aboutissent à la conclusion que, dès le Paléolithique inférieur, une certaine compétence opérationnelle est comparable à celle de l'homme moderne, en contexte technique. Les conclusions de M. Maingeot [1999] sur les techniques de pêche vont dans le même sens. B. Hayden [1993] estime, après P. Mellars [1989], et P.G. Chase, H. Dibble [1991] que l'homme de Néandertal n'est pas réellement différent de nous en ce qui concerne à la fois l'aptitude à la culture et l'aptitude aux activités symboliques. Pour ces auteurs, il est donc légitime d'employer des modèles cognitifs proches voire similaires à ceux utilisés pour l'homme moderne. Pour P. Mellars, en effet, il est maintenant certain que les dernières populations de Néandertal d'Europe de l'Ouest se comportaient exactement comme au paléolithique supérieur, au moins en ce qui concerne l'expression cognitive ou symbolique. J.-M.Geneste et M. Patou-Mathis pensent de même que Néandertal manipulait régulièrement et habilement concepts et symboles, et que les différences relatives de la taille du cerveau ou de sa morphologie ne révèlent rien d'important. Les auteurs statuent que les différences cognitives entre populations du paléolithique moyen et supérieur sont légères. Quant à L. Schepartz [1993], il estime qu'il n'y a aucune différence entre la cognition contemporaine et celle des néandertaliens.

Pour T.Wynn, les premiers comportements cognitifs spécifiquement humains apparaissent avec le biface. Il y voit la marque de capacités cognitives bien supérieures à celles de la culture des galets aménagés. T. Wynn [1993 a, b] s'intéresse aux capacités cognitives en jeu dans la perception de l'espace, à l'aide de l'épistémologie génétique de J. Piaget [1970]. Il en conclut que les tailleurs hominidés n'étaient pas significativement moins intelligents que des adultes modernes. Pour lui, les activités de ces individus démontrent qu'ils avaient atteint le stade de «l'intelligence opérationnelle » de J. Piaget, ce qui lui permet aussi d'avancer l'hypothèse de l'existence d'un univers culturel, voire même d'éventuelles cosmogonies. Il conclut que l'intelligence moderne était pour ainsi dire atteinte il y a 300.000 ans.

Les capacités cognitives des hommes du Paléolithique moyen sont très voisines des nôtres sur des aspects complexes tels que stratégies de subsistance et technologie. Il apparaît donc tout à fait fondé d'utiliser, en toute connaissance de cause, des outils d'analyse développés pour l'homme contemporain. En effet, les méthodes de la psychologie cognitive que nous adoptons ici n'impliquent qu'un degré minimum de complexité cognitive du sujet étudié. Elles offrent la possibilité d'établir une description 
rigoureuse de l'organisation mentale, de façon relativement indépendante de l'espèce humaine considérée, et donc de ses capacités.

\section{LES ACTIVITÉS MENTALES FINALISÉES}

Les activités cognitives finalisées sont les activités cognitives qui sous-tendent la réalisation d'une tâche par un sujet. Elles sont orientées par des objectifs, et reposent sur une représentation de la situation par le sujet. Les informations spécifiques aux activités mentales finalisées sont le résultat des traitements sensoriels : identification d'objets, de positions, de mouvements, de significations. Ainsi définies, les activités cognitives finalisées ne sont pas l'apanage du genre Homo [J. Vauclair, 2002]. Leur modélisation est légitime indépendamment de l'espèce considérée.

Le comportement de subsistance des hommes du paléolithique décrit leurs activités d'exploitation des matières premières animales, végétales ou minérales, à toutes fins essentielles à la survie. Il est donc bien plus large que le comportement alimentaire, avec lequel il est parfois improprement confondu. Nous nous intéressons ici à l'exploitation de l'animal dont nous verrons qu'elle est motivée par de nombreux buts différents.

Le comportement de subsistance des hommes du paléolithique moyen est typiquement une activité mentale finalisée : il sous-tend la réalisation de tâches (acquisition et traitement) ; il est orienté par des objectifs (d'alimentation, d'habitat...) ; il repose sur l'utilisation préalable d'informations postérieures au traitement sensoriel (identifications d'animaux, de lieux, de circonstances, de significations éthologiques, climatiques, sociales...).

Nos connaissances sur les objets s'expriment par des catégories et par l'activité de catégorisation. Catégoriser, c'est délimiter une classe d'objets, et définir les liens de cette classe avec d'autres classes. Il s'agit d'un mécanisme autonome du cerveau des vertébrés qui ne repose ni sur le langage, ni sur la conscience. Comme les activités mentales finalisées, l'étude de la catégorisation est donc légitime pour toute espèce de primate. Nous proposons ici une méthode de modélisation des catégorisations mobilisées par l'homme du Paléolithique : nous verrons que la décomposition de certaines étapes du comportement de subsistance en étapes élémentaires autorise une interprétation rigoureuse de ce comportement en termes de catégories.

\section{RÉSEAU SÉMANTIQUE À BASE DE PROPRIÉTÉS}

Les catégories sont constituées par des ensembles de propriétés liées entre elles, que délimitent un ensemble d'objets possédant ces propriétés. A.M. Collins et M.R. Quillian ont proposé, en 1969, que l'organisation des connaissances en mémoire pouvait être représentée graphiquement par un réseau sémantique. Un réseau comporte des nœuds et des arcs. Les nœuds du réseau sont les concepts, les arcs sont les relations entre 
concepts. À chaque nœud sont stockées les propriétés spécifiques d'un concept, tandis que les propriétés plus générales sont stockées à un niveau superordonné dans le réseau. Par exemple la propriété «a des ailes » est stockée au niveau de « oiseau » alors que « se déplace » l'est au niveau de « animal ».

Or, pour S. Poitrenaud [1998, 2001], les connaissances sur l'action associée à des objets sont structurées comme les connaissances sur ces objets. Il est donc possible de définir un réseau sémantique des actions, au même titre que le réseau sémantique des objets. Dans ce cadre, l'équivalent de la relation «catégorie - exemplaire » est la relation "procédure de réalisation - processus de réalisation ». Par procédure, il faut entendre l'équivalent d'une recette, alors que le processus serait constitué d'une réalisation particulière de cette recette. Ce point de vue est du plus haut intérêt pour le préhistorien qui dispose souvent de données de type «résultat - procédure de réalisation », en particulier dans l'étude du comportement de subsistance. En considérant comme «procédure de réalisation » le but des hommes du Paléolithique lors des activités de subsistance, par exemple l'alimentation ou la recherche de fourrure, et comme «processus de réalisation » la séquence d'opérations de boucherie observée sur le site, on voit qu'il est possible d'établir une très forte corrélation entre l'approche palethnologique et la démarche psycho-cognitive.

Pour un réseau sémantique d'actions, les nœuds sont les catégories d'objets, tandis que les arcs représentent la relation entre ces catégories. L'intérêt de cette approche pour la préhistoire est que ce sont les connaissances procédurales, les savoir-faire, qui sont considérés comme des propriétés des objets, ces savoir-faire étant souvent identifiables dans le cadre du comportement de subsistance au Paléolithique [M. Patou-Mathis 1996, 1997].

De ce point de vue, le comportement de subsistance doit faire apparaître des objets définis par les procédures qui leur sont applicables autant que par leurs propriétés de surface. Un stylo, par exemple, est défini autant par sa forme et sa taille (propriétés de surface) que par le fait qu'il permette d'écrire, qu'il puisse être tenu entre les doigts, qu'il permette de pointer, qui sont des procédures (propriétés fonctionnelles).

\section{OBJETS ET PROPRIÉTÉS DU COMPORTEMENT DE SUBSISTANCE}

L'étude du comportement de subsistance s'appuie essentiellement sur les traces d'utilisation de l'animal, pour lesquelles on dispose d'un grand nombre d'informations. La richesse des informations apportées par l'analyse du comportement de subsistance permet d'établir des hypothèses sur le comportement détaillé des hommes du Paléolithique.

Les études archéozoologiques ont permis de définir le concept de chaîne opératoire d'acquisition et de traitement des animaux par les hommes préhistoriques [M. Patou-Mathis, 1993]. En effet, il est possible de décomposer le traitement de l'animal en une séquence d'étapes techniques de boucherie. Un grand mammifère recèle 
de nombreuses ressources alimentaires et aussi non alimentaires : il peut être considéré comme un ensemble de matières premières.

Ces opérations conduisent à la récupération de différentes matières premières : nous retiendrons ici : viande, tendons, moelle, graisse, os, viscères, bois animal. Ces matières premières sont des objets qui peuvent être associés par les paléolithiques à des buts variés. Il a été possible de déterminer de nombreux buts effectivement poursuivis par ces hommes. Ces buts constituent du point de vue cognitif des propriétés des objets préalablement définis.

Il est possible de présenter les liens entre les objets et leurs propriétés sous forme matricielle, dans un tableau. Quand un objet (une matière première) possède une propriété (est affectée à un but), on coche la cellule d'intersection. Des tableaux de ce type ont été analysés à l'aide du logiciel STONE, développé par S. Poitrenaud, de l'Université Paris 8-Saint Denis. STONE restitue les relations à l'œuvre dans le tableau, en les présentant sous la forme graphique d'un treillis de Galois, Galois étant le mathématicien à l'origine de la procédure utilisée dans le logiciel. Mathématiquement, le treillis de Galois est une structure algébrique qui formalise les relations entre catégories sous-tendues par le tableau initial à double entrée [M. Barbut, B. Montjardet, 1970] : l'ensemble des couples objets - propriétés forme une relation binaire et le treillis est engendré à partir du calcul de toutes les combinaisons contenues dans cette relation. Les relations entre catégories sont dites d'implication, car elles imposent une hiérarchie aux catégories d'entrée. Cette hiérarchie distribue les catégories sur une échelle de généralité - spécificité.

Le diagramme est décrit selon des paramètres quantitatifs tels que le nombre de nœuds et d'arcs, le nombre de voisins de chaque catégorie et le nombre de niveaux d'abstraction, qui correspond au nombre maximum de nœuds rencontrés sur une lignée d'arcs orientés. Nous avons appelé «complexité cognitive» la mesure de ces grandeurs numériques, lui assignant une définition moins stricte que celle de $\mathrm{S}$. Poitrenaud, mais très fonctionnelle. Le graphe peut également être décrit d'après le positionnement relatif des catégories entre elles. La prise en compte des caractéristiques quantitatives et qualitatives du graphe permet d'appréhender la fluidité cognitive associée à la tâche sur le site. La notion de fluidité cognitive est ici employée au sens de D. Hofstadter [1995] : cet auteur suggère que l'analogie soit considérée comme une manifestation de la fluidité des concepts. Faire une analogie, c'est voir une situation nouvelle dans les termes d'une situation connue, au prix d'un glissement conceptuel. Ce glissement conceptuel est rendu possible par la caractéristique de « fluidité cognitive ».

\section{RÉSULTATS PRÉLIMINAIRES : UNE APPLICATION AU SITE DE TOURNAL À BIZE (AUDE)}

Ce niveau magdalénien ancien correspond à un habitat de chasseurs de rennes. Notre choix s'est porté sur ce site en raison de l'abondance de son matériel faunique et de sa bonne conservation. La richesse des données archéozoologiques a permis une 
interprétation palethnologique fournie et précise [M. Patou-Mathis et al., 1999 ; M. Patou-Mathis, 1997]. Quelques données non exhaustives sont brièvement rappelées ici. 8277 restes osseux ont été retrouvés, plus de $90 \%$ correspondant à du renne. 247 os de renne portent des marques d'origine anthropique, notamment de désarticulation. Des stries sont observées sur 87 os, dont $43 \%$ sur des tarsiens et métatarsiens. Dépouillement, décharnement et raclage sont attestés par leurs stries caractéristiques, de même que le prélèvement des tendons et diverses utilisations des os comme matière première. 25 os de bovinés et équidés attestent de leur traitement par l'homme, ainsi que quelques autres espèces.

Examinons maintenant le treillis de Galois établi à partir des données palethnologiques.

Tableau 1. Tableau des données palethnologiques en relations binaires

\begin{tabular}{|l|c|c|c|c|c|c|}
\hline & peau/tendons & viande & graisse/moelle & placenta & os & bois/dents \\
\hline Sert Pour art/parure & & & & & $\mathrm{X}$ & $\mathrm{X}$ \\
Se Mange & & $\mathrm{X}$ & & $\mathrm{X}$ & & \\
Sert d'outil & $\mathrm{X}$ & & & & $\mathrm{X}$ & $\mathrm{X}$ \\
Se conserve & $\mathrm{X}$ & $\mathrm{X}$ & & & & \\
Se prévoit & & & & $\mathrm{X}$ & $\mathrm{X}$ \\
\hline
\end{tabular}

\section{DESCRIPTION}

Les graphes (Figures 1 et 2) présentent des arcs orientés (présence d'une flèche) et des nœuds commentés. Un ensemble de plusieurs arcs bout à bout selon l'orientation des flèches s'appelle une lignée. Les nœuds du graphe présentent des objets (o pour object : objet) et les propriétés (f pour function : but) qui leur sont associées. Le graphe a la structure visuelle d'un treillis, et il est affiché par STONE de sorte que ses «branches » (les arcs) se croisent le moins possible. Mais cette contrainte n'est pas suffisante pour une lisibilité optimale, et nous utilisons une fonctionnalité supplémentaire du logiciel qui est de permettre de déplacer les nœuds à la souris. Bien entendu, lors de cette opération, les arcs restent indissociables de leur(s) extrémité(s), c'est-à-dire le ou les nœuds qu'ils incluent. En redisposant ainsi l'arbre initial, on rend visuellement plus lisibles le sens de l'inclusion de classe (alignement vertical des flèches) ainsi que le nombre de niveaux d'abstraction, c'est-à-dire le nombre maximum de nœuds traversé par une lignée. En effet, l'orientation retenue et la visibilité du niveau de profondeur facilitent l'analyse du graphe par ses objets, par ses propriétés, et par les catégories définies à chaque nœud.

La Figure 1 représente le graphe du treillis de Galois associé au Tableau 1. Elle décrit les classes de manières exhaustives et fait notamment apparaître les sommets du treillis. On peut y lire la composition des catégories (quels objets, associés à quelles 
propriétés) et leur structuration (graphe orienté avec structure d'inclusion de classes).

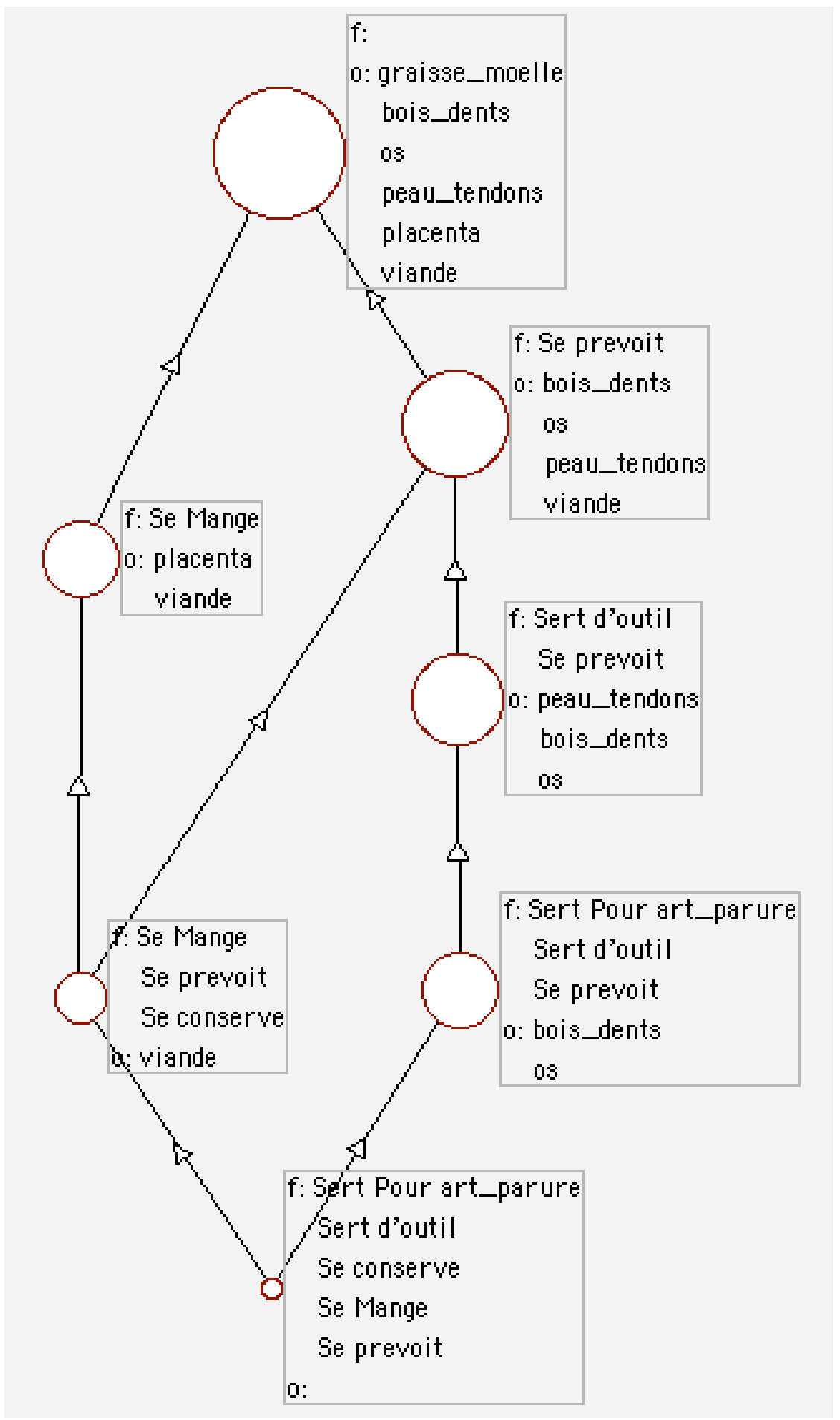

Figure 1. le graphe du treillis de Galois associé à la tâche

Les 2 sommets du graphe ont ici un statut particulier: les éléments de la classe superordonnée, qui regroupe l'ensemble des objets, ne sont associés à aucune propriété, 
autrement dit aucun but commun n'est réalisé sur l'ensemble des objets. De manière duale, la classe subordonnée, constituée de l'ensemble de propriétés, ne contient aucun objet, autrement dit il n'y a aucun objet sur lequel l'ensemble des buts soit réalisé. Le graphe illustre également la relation entre l'intension et l'extension d'une classe. Plus l'intension (les propriétés) est grande, plus l'extension (les objets) est réduite et viceversa. Par exemple la classe (se prévoit; bois_dent, os, peau_tendons, viande) a son intension réduite à une propriété (se prévoit) et une extension de 4 objets (bois_dent, os, peau_tendons, viande) alors que la classe (Sert pour art_parure, Sert d'outil, Se prévoit ; bois_dent, os) a une intension plus large (Sert pour art_parure, Sert d'outil, Se prévoit ) qui contient la précédente et une extension plus réduite (bois_dent, os) qui est contenue dans la précédente.

La Figure 2 constitue une description du même graphe (et du même Tableau) sur laquelle des transformations ont été opérées afin d'accroître l'intelligibilité du graphe dans l'optique d'interprétations psychologiques. Les 2 sommets du graphe ont été supprimés car ils n'ont pas de pertinence psychologique : rien ne justifie de regrouper l'ensemble des objets du fait qu'ils n'ont aucune propriété en commun, ni de regrouper l'ensemble des propriétés du fait qu'elles ne s'appliquent dans leur ensemble à aucun objet; ces classes ne sont pas susceptibles de constituer des catégories. Outre cette modification, le graphe est présenté de manière décumulée afin qu'il ne soit pas redondant et que la spécificité de chaque classe, qui constitue sa justification psychologique, apparaisse explicitement; les mécanismes d'héritage de propriétés et d'inclusion de classe permettant de reconstruire aisément les extensions et les intensions

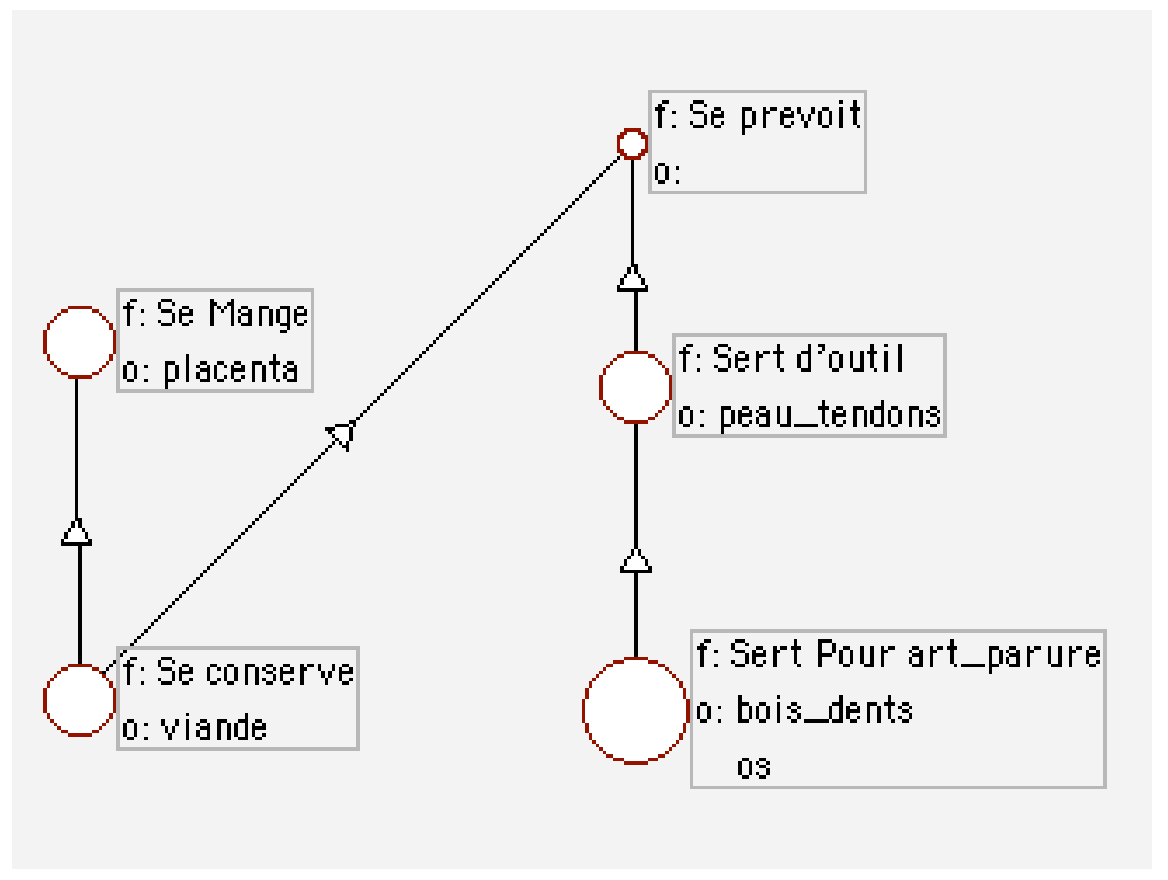

Figure 2. Le graphe simplifié du treillis de Galois associé à la tâche

de chaque classe. Ainsi on peut lire Figure 2 que la propriété «Sert pour art_parure » est spécifique aux objets «bois_dents» et «os », sachant également que ces objets héritent 
des propriétés des classes superordonnées (ici, «Sert d'outil» et « Se prévoit ») et que, de manière duale, les objets «bois_dents» et «os» font partie de la classe des objets ayant les propriétés «Sert d'outil » et «Se prévoit». Du fait de la visée de cette modélisation, qui est de traduire des mécanismes cognitifs, on privilégiera la présentation de la Figure 2, sachant qu'il est toujours possible, et de manière univoque, de passer d'une description à une autre, à la restriction près des objets n'ayant aucune propriété (c'est la cas ici du placenta) ou des propriétés qui ne sont applicables à aucun objet, et qui n'apparaissent pas dans le graphe simplifié, tout comme les sommets lorsqu'une des intersections est vide. D'un point de vue psychologique, chaque nœud du réseau est un ensemble objet - propriétés : il s'agit d'une catégorie, telle qu'elle a été définie précédemment. Le treillis présente donc non seulement les catégories cognitives à l'œuvre dans le comportement de subsistance, mais aussi leurs relations : on assiste à l'opération cognitive de catégorisation. Une catégorie est d'autant plus générale qu'elle est placée topographiquement loin de la source de sa lignée orientée. Plus sa source est distante, plus elle présente sur le graphe une forte extension : beaucoup d'objets s'y rapportent. Il s'agit d'une catégorie superordonnée. Les catégories sont d'autant plus spécifiques qu'elles sont situées topographiquement près de la source de la lignée orientée. Plus elles se trouvent proches de la source de la lignée, plus elles présentent sur le graphe une intension (ou compréhension) élevée, c'est-à-dire que beaucoup de propriétés s'y rapportent. Il s'agit de catégories subordonnées.

Les résultats palethnologiques sont de très haute complexité pour ce site : ils expriment directement les procédures et les buts ayant été appliqués aux parties exploitables des rennes. Les buts sont les buts ultimes, de haut niveau cognitif : art/parure, prévision et conservation en particulier.

Quelques regroupements d'objets ont été effectués pour s'adapter aux données palethnologiques. Ces regroupements cumulent les propriétés des objets individuellement considérés. Ainsi, les super-objets peau/tendons, graisse/moelle et bois/dents présentent chacun au moins une propriété d'un de leurs objets constitutifs, et pas seulement celles qu'ils partagent tous deux : nous avons réalisé la réunion des propriétés, non leur intersection.

La propriété « Se prévoit » a été réservée à la planification de l'acquisition, c'està-dire le choix du site de campement en fonction des critères cynégétiques, et à la chasse elle-même. Pour associer cette procédure aux objets concernés, nous sommes partis du principe que les parties exploitables de l'animal qui n'ont pas été recherchées au moment du traitement ne l'ont pas non plus été au moment de la planification de la chasse.

Par exemple, la graisse et la moelle n'ont pas été exploitées et nous avons donc considéré que cette ressource n'a pas non plus été convoitée au moment de planifier l'acquisition puis de la réaliser. En revanche, le cas contraire se présente : d'après les résultats palethnologiques concernant ce niveau d'occupation, se fondant eux-mêmes sur l'étude de la structure de la population de rennes abattus [L. Fontana, 1998], il semblerait que la chasse aux femelles gravides ait été effectuée, mais non 
systématiquement: la chasse apparaît comme non sélective, il est constaté « une similarité avec le profil de la population vivante de référence » [M. Patou-Mathis, 1994]. L'absence de lien entre la procédure « se prévoit» et le placenta s'explique donc par le fait que cette partie exploitable ne semble pas avoir été recherchée systématiquement pour elle-même.

\section{INTERPRÉTATION}

Nombre de catégories : 5

Nombre de relations d'implication : 4

Nombre de niveaux d'abstraction : 3

Chaque procédure est associée à une catégorie individuelle. Deux catégories sur cinq sont liées à une seule voisine sur le réseau, tandis que les trois autres sont liées à deux voisines immédiates. Ces paramètres d'assez faible amplitude suggèrent que pour les procédures choisies dans le tableau, la fluidité cognitive reste assez faible, ce qui peut paraître surprenant. En effet, le nombre de procédures par catégorie d'une part et le nombre moyen de voisins immédiats d'autre part laissent peu de potentialités aux glissements de sens et par là même aux mécanismes cognitifs tels que l'analogie. Nous suggérons que cette particularité pourrait s'expliquer du fait que trois des cinq procédures d'entrée du tableau initial relèvent d'une complexité cognitive déjà forte : «Se prévoit », «Se conserve » et «Sert pour Parure » sont toutes trois des procédures de haut niveau. En considérant de telles facultés comme briques élémentaires du tableau (comme du treillis), la fluidité cognitive peut être «comprise » à l'intérieur des procédures d'entrée, mais pas visible directement.

La catégorie superordonnée du graphe est la catégorie de prévision au sens préalablement défini (planification de l'acquisition et acquisition). C'est elle qui comporte le maximum d'objets. Elle correspond en quelque sorte à l'image principale que représente le renne pour le chasseur, comme source de matières premières vitales ou secondaires. Elle présente la plus forte extension.

Les procédures subordonnées du treillis sont, quelle que soit la version retenue, «Sert Pour Art » et «Se Conserve »: très spécifiques, elles ne concernent qu'un petit nombre d'objets. Nous proposons d'interpréter leur position topographique remarquable sur le réseau sémantique d'actions comme un signe possible de l'apparition tardive de ces procédures par rapport à celles de l'alimentation ou de la prévision, au cours du processus d'hominisation. En effet, la procédure de prévision telle qu'elle a été définie semble moins complexe que celles de conservation ou d'art et parures : liée strictement à la chasse et à son organisation, il peut sembler probable qu'elle ait pu être mise en œuvre relativement tôt lors de l'hominisation, ce qui est moins probable dans le cas du stockage de nourriture ou des manifestations artistiques. Nous suggérons que le treillis formalise ces relations. 
La catégorie « alimentation » est très peu liée à ses voisines : nous suggérons que sa position topographique illustre le fait qu'elle constitue un objectif prioritaire de la tâche de traitement, prioritaire étant ici à entendre au sens de la survie immédiate.

La catégorie des « outils », intermédiaire au sein de la hiérarchie général spécifique, liée à ses voisines, semble être la plus apte à favoriser les glissements de sens, et par conséquent des mécanismes cognitifs tels que l'analogie. La fluidité cognitive de la tâche de traitement, c'est-à-dire la souplesse, la mobilité cognitive d'un concept à l'autre, semble être plus particulièrement liée à la fabrication des outils.

\section{DISCUSSION}

Les propriétés qui nous intéressent sont des procédures, c'est-à-dire des actions possibles sur les objets. Or, en archéologie, les procédures sont bien entendu perdues, dans la mesure où il n'est pas possible de les observer directement. Une singularité essentielle de notre démarche intervient donc ici : la détermination des propriétés des objets, pour chaque tableau, fait intervenir une interprétation, celle du préhistorien. C'est le chercheur qui infère des données du terrain, que les paléolithiques ont coupé la viande ou brisé les os pour en extraire la moelle, par exemple. Ce qui est en cause ici n'est ni plus ni moins que le statut de vérité dans les sciences vouées à l'étude du passé.

La définition de certaines propriétés constitue un large consensus au sein des spécialistes. Certaines stries laissées sur des os longs, par exemple, sont la signature d'une décarnisation d'origine anthropique. Inférence au sens strict, cette affirmation, comme beaucoup d'autres en préhistoire, ne constitue pas moins un élément de base de la réflexion, à partir duquel des interprétations de deuxième niveau peuvent voir le jour. Il s'agit donc de ne pas perdre de vue cette introduction d'un ou deux niveaux interprétatifs au cœur même de la définition des propriétés des objets. Cette contrainte peut introduire des limites à l'interprétation de nos résultats.

Les idées de S. Mithen [1994, 1996a, 1996b] s'inspirent du modèle de fonctionnement cognitif proposé par J. Fodor [1986]. Les capacités cognitives seraient gérées par des «modules ». Ces modules seraient relativement indépendants au sein du cerveau, à l'origine, puis tendraient à interagir de plus en plus entre eux au fur et à mesure de l'hominisation. Le développement des capacités cognitives du Paléolithique à l'homme moderne correspondrait à cette intégration progressive du fonctionnement de tous les modules, et non à leur apparition. Pour l'auteur, l'intelligence néandertalienne était distribuée sur des modules relativement indépendants (domain-specific). Cette hypothèse pourrait être testée à l'aide des outils de la psychologie cognitive des actions finalisées, et en particulier du logiciel STONE, car l'analyse objet - propriétés facilite une comparaison rigoureuse entre groupes de données. 


\section{CONCLUSION}

Cette analyse de la catégorisation devrait permettre de dresser une carte cognitive de tout site de fouille suffisamment riche pour livrer des informations palethnographiques. Le réseau sémantique d'action associé au comportement des occupants paléolithiques pourrait faire l'objet d'un relevé systématique, au même titre que l'arsenal de données sédimentologiques, stratigraphiques, palynologiques, paléontologique ou lithiques qui caractérisent actuellement tout chantier. L'enregistrement des réseaux sémantiques d'action permettrait ultérieurement d'engager les premières comparaisons cognitives entre sites synchroniques ou diachroniques, et même, pourquoi pas ethnologiques, première ouverture rigoureuse au mystère de la paléocognition.

\section{BIBLIOGRAPHIE}

BARBUT M., MONJARDET B., Ordre et classification. Algèbre et combinatoire, tome 2, Paris, Hachette, série Hachette Université, 1970, p. 7-34.

BEDNARIK R. G., "Concept-mediated Marking in the Lower Paleolithic », Current Anthropology, 36 (4), 1995, p. 605-634.

BELFER-COHEN A., GOREN-INBAR N., «Cognition and communication in the Levantine Lower Paleolithic », World Archaeology, Vol. 26, 2, 1994, p. 144-157.

CHASE P.G., DIBBLE H., «Middle Paleolithic Symbolism », Journal of Anthropological Archaeology, 6, 1991, p. 263-296.

COLLINS A.M., QUILLIAN M.R., « Retreival Time from Semantic Memory », Journal of Verbal Learning And Verbal Behavior, 8, 1969, p. 240-247.

DUCROT O., TODOROV T., Dictionnaire encyclopédique des sciences du langage, Paris, Seuil, 1972, 470 p.

DUPUY J.P., Aux origines des sciences cognitives, Paris, La Découverte, 1994, 188 p.

FODOR J., La modularité de l'esprit, Paris, Minuit, 1986, 178 p.

FONTANA L., Mobilité et subsistance au Magdalénien dans le Languedoc occidental et le Roussillon, thèse de doctorat en Préhistoire, Ethnologie, Anthropologie, Université de Paris 1, 2 tomes, 1998.

GERVET J., GALlO A., CHALMEAU R., SOlEILHAVOUP M., "Some prerequisites for a study of the evolution of cognition in the animal kingdom », Acta Biotheorica, 44, 1996, p. 37-57.

HAYDEN B., "The cultural capacities of neanderthals: a review and re-evaluation », Journal of Human Evolution, 24, 1993, p. 113-46.

HOFSTADTER D., Fluid concepts and creative analogies, New York, Basic Book, $518 \mathrm{p}$.

KUHN S.L., "On planning and curated technologies in the Middle Paleolithic », Journal of Anthropological Research, 48(3), 1992, p. 185-214. 
MAINGEOT M., Préhistoire de la pêche aux paléolithique, épipaléolithique, mésolithique. Comparaisons ethnologiques et réflexions, Mémoire de l'Université de Liège, Belgique, 1999, 2 vol.

MELLARS P., "Major issues on the emergence of modern humans », Current Anthropology, 30(3), 1989, p. 332-335.

MITHEN S., «From domain-specific to generalised intelligence: A cognitive interpretation of the Middle/Upper Paleolithic transition », The Ancient Mind, C. Renfew, E. Zubrow eds., Cambridge, Cambridge University Press, 1994, p. 3-30.

MITHEN S., The prehistory of the mind: a search for the origins of art, religion, and science, London, Thames and Hudson, 1996(a), 288 p.

MITHEN S., « On Early Paleolithic 'Concept-mediated Marks', Mental Modularity, and the origin of Art », Current Anthropology 37(4), 1996(b), p. 666-670.

NOBLE W, DAVIDSON I, « The evolutionary emergence of modern human behaviour: Language and its archaeology », Man: Journal of the Royal Anthropological Institute, 26, 1991, p. 223-253.

OTTE M., Comportement de l'homme au moustérien, dossiers Histoire et Archéologie, 124, 1988.

OTTE M., PATOU-MATHIS M., « Comportements de subsistance au Paléolithique moyen en Europe. Les Eyzies », Paléo, 4, 1992, p. 29-34.

PATOU-MATHIS M., «Étude de la fracturation des os longs de renne ; méthode et résultats : le niveau Magdalénien 'archaïque' de la grotte Tournal à Bize (Aude)», in Bone Modification Conference, Hot Springs, South Dakota, USA, Archaeology Laboratory, Occasional Publication ${ }^{\circ}$ 1, 1993, p. 195-219.

PATOU-MATHIS M., «Archéozoologie des niveaux moustériens et aurignaciens de la grotte Tournal à Bize (Aude)», Gallia Préhistoire, 36, 1994, p. 1-64.

PATOU-MATHIS M., "Techniques d'acquisition et de traitement des grands mammifères par les néandertaliens européens : exemples de 'chaînes opératoires' », Quaternaria nova, VI, 1996, p. 187-203.

PATOU-MATHIS M., «Les marques de boucherie au Paléolihique », Revue Méd. Vét., 148 (12), 1997, p. 959-968.

PATOU-MATHIS M., BAYLE G., PALETTA C., «Étude archéozoologique du niveau magdalénien 'ancien' de la grotte Tournal à Bize (Aude) », Préhistoire Européenne, 14, 1999, p. 115-133.

PELEGRIN J., «A framework for analysing prehistoric stones tools manufacture and a tentative application to some early stone industry », The use of tools by humans and non-human primates, A. Berthelet, J. Chavaillon eds., Oxford, Clarendon Press, 1993, p. 301-317.

PELEGRIN J., KARLIN C., BODU P., «Chaînes opératoires : un outil pour le préhistorien », Technologie préhistorique, J. Tixier éd., Paris, CNRS, 1988, p. 55-62. 
PIAGET J., L'épistémologie génétique, Paris, Presses Universitaires de France, 1970, $126 \mathrm{p}$.

POITRENAUD S., «The PROCOPE semantic network: An alternative to action grammars », International Journal of Human-Computer Studies, 42, 1998, p. 31-69.

POITRENAUD S., Complexité cognitive des interactions Homme-Machine. Modélisation par la méthode ProcOpe, Paris, L'Harmattan, 2001, 220 p.

RICHARD J.-F., Les activités mentales, Paris, Armand Colin, 1990, 434 p.

SAILLOT I., OTTE M., PATOU-MATHIS M., « Une critique épistémologique des analyses de paléocognition », Préhistoire Européenne 16-17, 2000-2001, p. 9-15.

SCHEPARTZ L., «Language and modern human origin », Yearbook of Physical Anthropology, 36, 1993, p. 91-126.

VAUCLAIR J., « Categorization and conceptual behavior in non human primates », The Cognitive animal, M. Bekoff, C. Allen, G. Burghardt eds., Cambridge MA, MIT Press, 2002, p. 239-245.

WYNN T., « The Intelligence of Later Acheulean Hominids », Man, 14, 1979, p. 371391.

WYNN T., «The Intelligence of Oldowan Hominids », Journal of Human Evolution, 10, 1981, p. 529-541.

WYNN T., «Layers of thinking in tool behavior », Tools, language and cognition in Human Evolution, K. Gibson, T. Ingold eds., Cambridge, Cambridge Univ. Press, 1993(a), p. 389-406.

WYNN T., "Two Developments in the Mind of Early Homo », Journal of anthropological archaeology, 12, 1993(b), p. 299-322. 